\title{
Serotonin 2 Receptors, Agomelatine, and Behavioral and Psychological Symptoms of Dementia in Alzheimer's Disease
}

\author{
Hui-Hua Li $\mathbb{D},{ }^{1}$ Xiao-Yan Yao $\mathbb{D},{ }^{2}$ Sheng Tao $\mathbb{D},{ }^{1}$ Xue Sun $\mathbb{D}^{1}$, Pan-pan Li $\mathbb{D},{ }^{1}$ Xi-xin Li $\mathbb{D},{ }^{1}$ \\ Zhu-Li Liu iD, 2 and Chao Ren (D) $^{2}$ \\ ${ }^{1}$ Zhenjiang Mental Health Center, The Fifth People's Hospital of Zhenjiang City, Zhenjiang 212000, Jiangsu Province, China \\ ${ }^{2}$ Department of Neurology, The Affiliated Yantai Yuhuangding Hospital of Qingdao University, Yantai 264000, \\ Shandong Province, China
}

Correspondence should be addressed to Zhu-Li Liu; 13188769066@163.com and Chao Ren; renchaotg@126.com

Received 22 January 2021; Revised 16 March 2021; Accepted 24 March 2021; Published 31 March 2021

Academic Editor: Giuseppe Bellelli

Copyright (C) 2021 Hui-Hua Li et al. This is an open access article distributed under the Creative Commons Attribution License, which permits unrestricted use, distribution, and reproduction in any medium, provided the original work is properly cited.

\begin{abstract}
There are nearly 50 million Alzheimer's disease (AD) patients worldwide, 90\% of whom develop behavioral and psychological symptoms of dementia (BPSD), which increase the mortality rate of patients, and impose an economic and care burden on families and society. As a neurotransmitter and neuromodulator, serotonin is involved in the regulation of psychoemotional, sleep, and feeding functions. Accumulating data support the importance of serotonin in the occurrence and development of BPSD. Studies have shown that reduction of serotonin receptors can increase depression and mental symptoms in AD patients. At present, there is no drug treatment for AD approved by the US Food and Drug Administration. Among them, agomelatine, as a new type of antidepressant, can act on serotonin 2 receptors to improve symptoms such as depression and anxiety. At present, research on BPSD is still in the preliminary exploratory stage, and there are still a lot of unknowns. This review summarizes the relationship between serotonin 2 receptors, agomelatine, and BPSD. It provides a new idea for the study of the pathogenesis and treatment of BPSD.
\end{abstract}

\section{Introduction}

Alzheimer's disease (AD) is a major health burden. In 2016, the International Alzheimer's Disease Association pointed out that there are nearly 50 million AD patients worldwide, $90 \%$ of whom develop behavioral and psychological symptoms of dementia (BPSD). It is estimated that by 2050, a new case of AD will occur every $33 \mathrm{~s}$, and nearly 1 million new cases will appear every year. The number of global AD patients may reach 130 million $[1,2]$, which increases the mortality rate of patients, and imposes an economic and care burden on families and society [3]. AD is ranked fourth in the causes of death among elderly people. The mortality of AD patients with BPSD increases significantly [4]. Considering the safety of patients and others, as well as family members often unable to bear the care and financial burdens, the number of patient visits and hospitalizations is increasing, and even the survival rate is often reduced, which has attracted the attention of medical staff $[5,6]$. Therefore, it is urgent to find the pathogenesis and treatment of BPSD. However, a lot of research is still in the preliminary exploratory stage. Genetic studies have suggested that serotonin $2 \mathrm{~A}$ and $2 \mathrm{C}$ genes are likely susceptibility factors for various mental illnesses such as BPSD, schizophrenia, affective disorders, and anxiety [7, 8]. Agomelatine is a new type of antidepressant, a selective agonist of melatonergic MT1 and MT2 receptors and a serotonin 5-HT2C receptor antagonist [9]. Agomelatine has affinity for some members of the serotonin 2 subfamily [10]. In this report, we provide a review of the relationship between serotonin 2 receptors, agomelatine, and BPSD.

\section{Definition and Epidemiological Characteristics of Alzheimer's Disease (AD) and Behavioral and Psychological Symptoms of Dementia (BPSD)}

$\mathrm{AD}$, the most common cause of dementia, is a progressive neurological degenerative disease caused by a variety 
of biological, psychological, and social factors. Severe memory impairment and cognitive decline may occur, causing severe functional impairment to the patient, as well as imposing a heavy burden on the family and society. A systematic review and meta-regression analysis of dementia in China revealed that the prevalence of $\mathrm{AD}$ over the past 30 years (from 1985 to 2015) among people over 55 years of age was $4.03 \%$ [11]. Another epidemiological survey showed that in China, the prevalence of dementia among people aged $\geq 65$ years was $5.14 \%-$ $7.3 \%$, and it is estimated that there are about 8 million dementia patients in China [12]. Some researchers have calculated that the number of dementia patients in China will reach 22 million by 2040. Among them, the proportion with $\mathrm{AD}$ is the highest, accounting for $60-70 \%$ of all dementias [13].

The clinical manifestations of $\mathrm{AD}$ patients include the common cognitive symptoms such as nonrecognition and dyslexia, as well as noncognitive symptoms, which are becoming more important. They are generally called behavioral and psychological symptoms of dementia (BPSD) and are also known as neuropsychiatric symptoms. In 1996, the International Psychogeriatric Association defined BPSD as "a term used to describe a heterogeneous range of psychological reactions, psychiatric symptoms, and behaviors occurring in people with dementia of any etiology" [14]. Burns et al. [15-18] described BPSD in four areas: disorders of thought content (delusions, etc.), disorders of perception (misidentification syndromes, hallucinations, etc.), mood disorders (anxiety, depression, etc.), and behavior disorders (agitation, aggression, etc.). Xue and Zhang [19] summarize BPSD into eight major symptoms: delusions, hallucinations, affective disorders (anxiety, depression, apathy, irritability, etc.), aggressive behavior, abnormal activities, eating disorders, changes in biological rhythms, and sexual dysfunction. These symptoms can occur at any stage of the progression of $\mathrm{AD}$, and the symptoms and frequency can vary. According to epidemiological surveys, BPSD can occur in $>90 \%$ of $\mathrm{AD}$ patients [20]. In the early stage, $\mathrm{AD}$ patients may develop reactive depression and anxiety, and in the middle stage, symptoms such as aggression and sleep disturbance often appear. However, in the later stage, due to severe impairment of social functions, depression, emotional apathy, and emotional decline may occur. Studies have found that sleep disorders, irritability, and apathy are considered to be the most common BPSD in patients with $\mathrm{AD}$ in China. Among them, apathy is a negative symptom that shows a lack or suppression of passion, emotion, or excitement and is common in AD (70-90\%) [21]. Apathy can occur alone or in combination with depression and anxiety. Apathy is one of the most common neuropsychiatric symptoms. Multiple reports indicate that $>80 \%$ of $\mathrm{AD}$ patients have high levels of stress and half have depression and anxiety [22]. Depressive symptoms, as a common type of emotional disorders associated with dementia, have been reported differently. Burns et al. reported a frequency of $0-87 \%$, but most reports are between $14 \%$ and 29\% [23], and the Chinese literature reports that one-third of BPSD is accompanied by depressive symptoms.

\section{Serotonin 2 Receptors and Pathogenesis of BPSD}

At present, the research on BPSD is mainly based on the summary of clinical characteristics, and there are few reports on its pathogenesis. Therefore, further exploration of the pathogenesis of BPSD is beneficial to the choice of therapeutic drugs and the development of new drugs.

Serotonin, also known as 5-hydroxytryptamine (5-HT), is widely distributed in the central and peripheral nervous systems, mainly in the hippocampus, hypothalamus, and cortex. Forebrain serotonin is derived from dorsal raphe nucleus neurons of the midbrain and participates in the regulation of psychoemotional, sleep, and feeding functions. It is well known that serotonin dysfunction exists in $\mathrm{AD}$ patients, which is manifested in the reduction of serotonin and its metabolites in the brain of elderly $\mathrm{AD}$ patients, the loss of serotonin neurons in the raphe nucleus, and decreased serotonin $2 \mathrm{~A}$ receptors in the cortex and hippocampus [24-26]. The reduction of serotonin receptors may be negatively correlated with BPSD [2]. Serotoninergic neurotransmitters are jointly regulated by receptors of different affinity and functionality, as well as serotonin transporters. At present, there are seven serotonin receptor subtypes, and there are different research results in the correlation between each subtype and BPSD. Among them, serotonin 1A, 2A, 2C, 6, and 7 are thought to be related to the occurrence and development of various BPSD [2]. The human serotonin $2 \mathrm{~A}$ receptor is located on chromosome 13q14-q21 and has high sequence homology with serotonin 2C located at Xq24 [7]. The two receptors are highly conserved between humans, mice, and rats. The reduction of serotonin $2 \mathrm{~A}$ and $2 \mathrm{C}$ receptors and polymorphisms of the genes coding the two receptors are thought to be related to the occurrence of BPSD [26]. Pritchard et al. [7] support a role of 5-HT2A T102C in psychotic symptoms and a role for 5 -HT2C cys 23 ser in anxiety and appetite disturbance. However, the results of human research vary. Some studies have shown that serotonin $2 \mathrm{~A}$ is associated with an increase in mental illnesses such as depression, while others have shown no relationship and similar findings in serotonin $2 \mathrm{C}[8,27,28]$. For example, Japanese researchers have found that Yokukansan (a traditional Japanese herbmedicine) can relieve the head-twitch response (an indicator of hallucination-like symptoms) in the mouse model by mediating downregulation of serotonin $2 \mathrm{~A}$ receptors in the prefrontal cortex [29]. Shimizu et al. [30] believe that the decrease in serotonin $2 \mathrm{~A}$ and $2 \mathrm{C}$ receptors may be related to the occurrence of BPSD in patients with AD. Holmes et al. [28] found that $\mathrm{AD}$ patients with different serotonin $2 \mathrm{~A}$ and $2 \mathrm{C}$ receptor genotypes have a significantly increased risk of depression. However, Pockros et al. [31] believe that the serotonin $2 \mathrm{~A}$ receptor antagonist and the serotonin $2 \mathrm{C}$ receptor agonist can reduce neural excitability and reduce hyperlocomotion, both of which mediate opposing behavioral effects.

Taken together, these studies indicate that the serotoninergic neurotransmitter system is a target for susceptibility to $\mathrm{AD}$ and BPSD (Figure 1). The role of these two serotonin 2 receptor subtypes in BPSD needs further study. The 


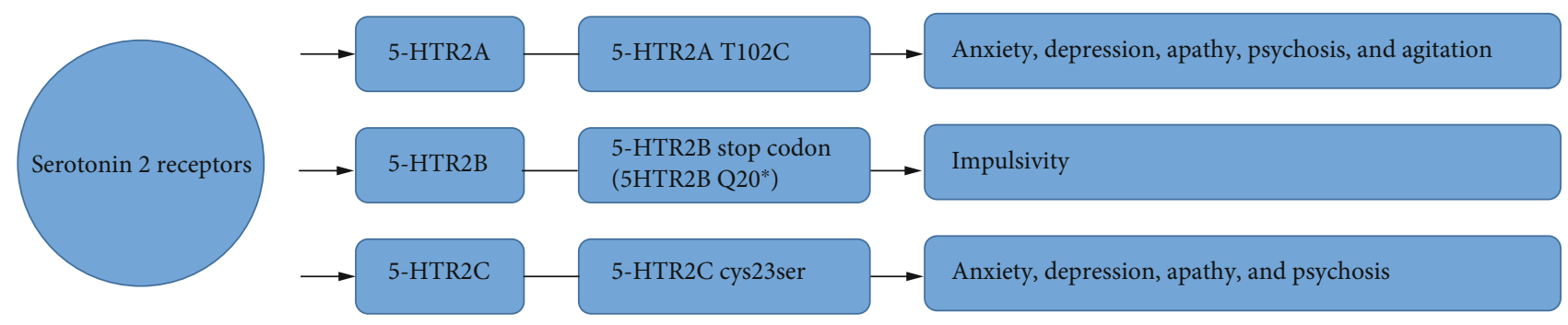

FIGURE 1: The relationship between 5-HT receptor subtypes and genotypes and BPSD.

relationship between serotonin $2 \mathrm{~A}$ and $2 \mathrm{C}$ receptors, and whether they interact with each other, is an important research topic. This may suggest a new approach to alleviate the symptoms of mental behavior.

\section{Treatment of BPSD}

The treatment of BPSD in AD patients is currently in the exploratory stage. Although BPSD severely affects the quality of life of elderly people with $\mathrm{AD}$ and their families, there is no unified and effective drug to control the progress of $\mathrm{AD}$ cognitive impairment. However, compared with the cognitive dysfunction of $\mathrm{AD}$, we can better control BPSD through appropriate psychiatric intervention and treatment, improving patient symptoms and quality of life, and reducing the burden on caregivers.

At present, there are two main types of therapeutic schedules for BPSD: nonpharmacological and pharmacological interventions. Chinese guidelines for the diagnosis and treatment of dementia and cognitive impairment recommend nonpharmacological treatments as a first-line treatment for BPSD, including music therapy, cognitive behavioral therapy, physical therapy, etc. The memory therapy developed by Butleras has been reported to improve depressive symptoms in patients with BPSD [32], but its onset is often slower and is suitable for patients with mild disease. Therefore, in patients in whom nondrug treatment is not effective, drug intervention is needed. At present, the drug treatment of $\mathrm{AD}$ generally uses nootropic drugs. Acetylcholinesterase (ACHE) inhibitors (e.g., donepezil) and N-methyl-D-aspartic acid (NMDA) receptor antagonists (such as memantine) are representative [33]. For severe BPSD, additional psychotropic drugs are needed, including antidepressants, antipsychotics, anxiolytics, and sedative hypnotics.

With the deepening of research, it has been found that antidepressants may also play a role in the treatment of $\mathrm{AD}$ and BPSD. Although some antidepressants have been applied to adjuvant treatment of AD and BPSD in clinical practice and proved to be effective, the effectiveness of various antidepressants is not the same. Based on evidence-based medicine and the National Institute of Health and Clinical Optimization (NICE) clinical treatment guidelines, Jeyapaul et al. proposed that for the treatment of BPSD in AD patients, serotonin reuptake inhibitors (SSRIs) are preferred for depression. Sertraline or citalopram are the preferred SSRIs [34]. Studies have mostly used citalopram for research. It is reported that taking $30 \mathrm{mg} /$ day citalopram has a positive effect on agitation in dementia. Unfortunately, this study also confirmed the risk of causing QT extension [35]. Tricyclic and tetracyclic antidepressants have many adverse reactions and should be used with caution in elderly patients. Venlafaxine is an antidepressant in the class of selective serotonin-norepinephrine reuptake inhibitors. It has a quick onset and few adverse reactions, so it can be used as appropriate. Studies have shown that new antidepressants may be most helpful in the treatment of restlessness, while depression, apathy, and anxiety are worse in dementia $[33,36]$. As a new antidepressant, the application of agomelatine in AD and BPSD is worth exploring. Some experiments show that agomelatine have an anti-AD effect through repairing cholinergic system dysfunction, serotonergic system dysfunction, and glutamate/GABA system dysfunction; eliminating the $\beta$-amyloid protein; and inhibiting oxidative stress on multiple targets and multiple pathways. And there is also evidence that agomelatine has a unique pharmacological effect and improves depression, depressive symptoms, and sleep cycles in patients with AD [37]. Agomelatine can improve apathy in frontotemporal dementia [38]. Yao et al. [37] presented that agomelatine could prevent $\mathrm{A} \beta$ induced tau phosphorylation and oxidative damage in PC12 cells; agomelatine exerts neuroprotection in AD. So, its application in BPSD is worthy of clinical attention in the future.

Clinical studies have shown that the use of antipsychotic drugs can reduce BPSD, especially second-generation antipsychotics (SGAs) such as clozapine, risperidone, olanzapine, and quetiapine. SGAs work by blocking the serotonin receptor and dopamine receptor pathways and alleviating delusions, hallucinations, agitation, and aggressive behavior. Risperidone is approved for the treatment of AD. However, most literature also points out that there are safety issues in the treatment of BPSD with antipsychotic drugs, which have increased adverse events such as long-term tardive dyskinesia, cardiovascular and cerebrovascular accidents, and liver and kidney damage in AD patients.

As a result, the US Food and Drug Administration issued a black box warning that the use of antipsychotic drugs in patients with dementia increases the risk of death. The use of benzodiazepines and mood stabilizers to treat BPSD in patients with dementia is currently not recommended. This is because published data indicate a high risk of adverse reactions and a low probability of effective treatment [39].

\section{Possible Mechanism of Interaction between Agomelatine, Serotonin, and BPSD}

Treatment of AD is mainly to delay disease progression, and treatment of BPSD is to improve symptoms and reduce the 


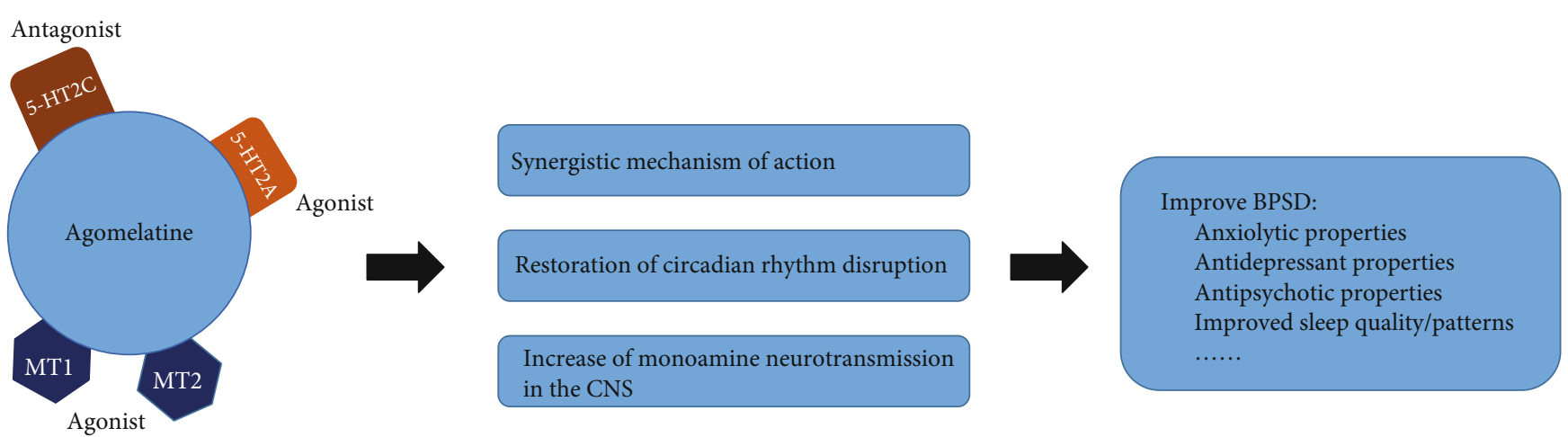

FIgURE 2: The possible mechanism of agomelatine to improve BPSD.

burden on caregivers. At present, the treatment of BPSD has become an important medical challenge for elderly patients. The two major pathological features of $\mathrm{AD}$ are the deposition of extracellular amyloid-beta $(\mathrm{A} \beta)$ peptides in the brain, leading to the formation of amyloid plaques and excessive phosphorylation of Tau protein to form neurofibrillary tangles. Studies have found that serotonin receptors affect the two major pathological features of AD. For example, AD mouse models that overexpress $\mathrm{A} \beta$ indicate that the pathological accumulation of $\mathrm{A} \beta$ depends on the reduction of serotonin 2A receptor expression [40]. Antagonizing 5-HTR2C can prevent tau hyperphosphorylation and enhance memory [41]. Studies have shown that melatonin and indole compounds (mainly serotonin) can inhibit and destabilize $\mathrm{A} \beta$ fibril formation, increase cell viability by $9-25 \%$, and have neuroprotective effects [42]. The serotonin 2 receptor family has become the developmental target of pharmacological treatments for a variety of mental disorders, including depression, schizophrenia, and addiction.

The new-generation antidepressant agomelatine has unique pharmacological properties and mechanism of action. Most studies have shown that agomelatine is a postsynaptic serotonin $2 \mathrm{C}$ receptor antagonist and melatonin receptor agonist [9]. It acts on the suprachiasmatic nucleus, hippocampus, frontal cortex, and striatum, leading to improved sleep duration, recovery of circadian rhythm, and improved mood. It has a positive effect on anxiety and depression. However, the drug does not adversely affect memory and attention during treatment, especially in elderly patients; does not affect blood pressure and heart rate; and does not cause habitual and withdrawal symptoms and behavioral toxicity [43]. At the same time, it can increase noradrenergic and dopaminergic neurotransmissions [44]. The serotonin is also a precursor of pineal melatonin; Zhou et al. found that melatonin was significantly reduced in cerebrospinal fluid samples from AD. In order to find new drugs for the treatment of $\mathrm{AD}$, a large-scale study that analyzes and predicts the global database of $\mathrm{AD}$ treatment drugs showed that agomelatine can interact with nine $\mathrm{AD}$-related targets (serotonin 2A receptor, ADORA2A, ACHE, BACE1, PTGS2, GABRG1, MAOB, SIGMAR1, and ESR1) to treat AD by repairing the serotonin, GABA, and cholinergic system [45]. This is different from the previous thought that agomelatine acts on the serotonin $2 \mathrm{C}$ receptor. Agomelatine could improve apathy and depression [33]. In 2016, researchers reported a case report. A 91-year-old AD patient with sleep disorders and depression symptoms improved significantly after using agomelatine [46]. Treatment of insomnia and depression in $\mathrm{AD}$ patients also has a positive effect on cognitive function. It provides a new direction for us to study the treatment of BPSD in AD patients. However, there are some reports that the use of drugs such as SSRIs and agomelatine can cause emotional deactivation in $\mathrm{AD}$ patients. Some researchers believe that this emotional disorder induced by $\mathrm{AD}$ is due to dopamine neurotransmission in the neural circuit that regulates reward processing downregulation and occurs secondary to the activation of serotonin $2 \mathrm{C}$ receptors in the nucleus accumbens [47].

However, the relationship between agomelatine and serotonin receptor and $\mathrm{AD}$ has been proven inconclusively. It is not clear what the specific relationship between agomelatine, serotonin receptor, and BPSD is. There are few reports on the relationship between the efficacy of agomelatine in the treatment of BPSD and serotonin receptors (especially considering both serotonin $2 \mathrm{~A}$ and $2 \mathrm{C}$ receptors). No significant changes in the two receptors following agomelatine have been reported.

\section{Discussion and Conclusion}

With the aging of society, the prevalence of $\mathrm{AD}$ is increasing rapidly. About $90 \%$ of people with dementia have at least one BPSD. These symptoms include depression, anxiety, and apathy. The improvement of these symptoms helps to improve the quality of life of patients. Therefore, it is urgent to find the pathogenesis and treatment of BPSD. However, the treatment of BPSD is currently in the preliminary exploratory stage. Many research results are also conflicting. This review attempts to find associations between serotonin, agomelatine, and BPSD. We put forward the following hypothesis: agomelatine can improve BPSD in $\mathrm{AD}$ patients and mainly works by acting on serotonin $2 \mathrm{~A}$ and $2 \mathrm{C}$ receptors, causing the serotonin concentration in the brain to increase and have changes in other monoamine transmitters. Thereby, it can further change mental symptoms, such as anxiety and depression (Figure 2). However, BPSD have a complex mechanism. This hypothesis also needs to be confirmed. The optimal drug therapy for BPSD is still under 
exploration. Antidepressants are currently being given high hopes, but the effects of antidepressants on BPSD vary considerably. Among them, agomelatine is a noteworthy candidate; accordingly, it can improve apathy, depression, anxiety, insomnia, etc. in BPSD. However, we should also be aware that everything has two sides; agomelatine also has its shortcomings. For instance, agomelatine has less evidence for efficacy in elderly patients, and the risk of liver damage may increase. This review is aimed at providing a new idea for the study of the pathogenesis and treatment of BPSD. We hope that in the future, we can clarify (1) what serotonin changes exist in patients with BPSD, (2) whether serotonin changes cause BPSD or become a marker for detecting BPSD, and (3) the link between BPSD and serotonin receptor subtypes.

\section{Consent}

Not applicable.

\section{Disclosure}

Hui-Hua Li and Xiao-Yan Yao are co-first authors.

\section{Conflicts of Interest}

The authors declare that they have no conflicts of interest.

\section{Acknowledgments}

This study was funded by the Zhenjiang Social Development Guiding Project (Grant no. FZ2019045).

\section{References}

[1] Alzheimer's Association, "2016 Alzheimer's disease facts and figures," Alzheimer's \& Dementia, vol. 12, no. 4, pp. 459-509, 2016.

[2] S. Chakraborty, J. C. Lennon, S. A. Malkaram, Y. Zeng, D. W. Fisher, and H. Dong, "Serotonergic system, cognition, and BPSD in Alzheimer's disease," Neuroscience Letters, vol. 704, pp. 36-44, 2019.

[3] L. Trillo, D. Das, W. Hsieh et al., "Ascending monoaminergic systems alterations in Alzheimer's disease. Translating basic science into clinical care," Neuroscience and Biobehavioral Reviews, vol. 37, no. 8, pp. 1363-1379, 2013.

[4] V. Bransvik, E. Granvik, L. Minthon, P. Nordstrom, and K. Nagga, "Mortality in patients with behavioural and psychological symptoms of dementia: a registry-based study," Aging Ment Health, vol. 18, pp. 1-9, 2020.

[5] J. Wancata, J. Windhaber, M. Krautgartner, and R. Alexandrowicz, "The consequences of non-cognitive symptoms of dementia in medical hospital departments," International Journal of Psychiatry in Medicine, vol. 33, no. 3, pp. 257-271, 2003.

[6] K. Yaffe, P. Fox, R. Newcomer et al., "Patient and caregiver characteristics and nursing home placement in patients with dementia," JAMA, vol. 287, no. 16, pp. 2090-2097, 2002.

[7] A. L. Pritchard, J. Harris, C. W. Pritchard et al., "Role of 5HT2A and 5HT2C polymorphisms in behavioural and psy- chological symptoms of Alzheimer's disease," Neurobiology of Aging, vol. 29, no. 3, pp. 341-347, 2008.

[8] L. Tang, Y. Wang, Y. Chen et al., "The Association between 5HT2A T102C and Behavioral and Psychological Symptoms of Dementia in Alzheimer's Disease: A Meta-Analysis," BioMed Research International, vol. 2017, Article ID 5320135, 14 pages, 2017.

[9] L. San and B. Arranz, "Agomelatine: a novel mechanism of antidepressant action involving the melatonergic and the serotonergic system," European Psychiatry, vol. 23, no. 6, pp. 396402, 2008.

[10] T. R. Norman and J. S. Olver, "Agomelatine for depression: expanding the horizons?," Expert Opinion on Pharmacother$a p y$, vol. 20, no. 6, pp. 647-656, 2019.

[11] Y. Zhu, H. Liu, X. L. Lu et al., "Prevalence of dementia in the People's Republic of China from 1985 to 2015: a systematic review and meta-regression analysis," BMC Public Health, vol. 19, no. 1, p. 578, 2019.

[12] J. Jia, F. Wang, C. Wei et al., "The prevalence of dementia in urban and rural areas of China," Alzheimers Dement, vol. 10, no. 1, pp. 1-9, 2014.

[13] S. A. Castro-Chavira, T. Fernandez, H. Nicolini, S. Diaz-Cintra, and R. A. Prado-Alcala, "Genetic markers in biological fluids for aging-related major neurocognitive disorder," Current Alzheimer Research, vol. 12, no. 3, pp. 200-209, 2015.

[14] C. H. lin and H. Y. Lane, "The role of N-methyl-D-aspartate receptor neurotransmission and precision medicine in behavioral and psychological symptoms of dementia," Frontiers in Pharmacology, vol. 10, p. 540, 2019.

[15] A. Burns, R. Jacoby, and R. Levy, "Psychiatric phenomena in Alzheimer's disease. I: disorders of thought content," The British Journal of Psychiatry, vol. 157, no. 1, pp. 72-76, 1990.

[16] A. Burns, R. Jacoby, and R. Levy, "Psychiatric phenomena in Alzheimer's disease. II: disorders of perception," The British Journal of Psychiatry, vol. 157, no. 1, pp. 76-81, 1990.

[17] A. Burns, R. Jacoby, and R. Levy, "Psychiatric phenomena in Alzheimer's disease. III: disorders of mood," The British Journal of Psychiatry, vol. 157, no. 1, pp. 81-86, 1990.

[18] A. Burns, R. Jacoby, and R. Levy, "Psychiatric phenomena in Alzheimer's disease. IV: disorders of behaviour," The British Journal of Psychiatry, vol. 157, no. 1, pp. 86-94, 1990.

[19] H. B. Xue and M. Y. Zhang, Treatment for Behavioral and Psychiatric Symptoms of Alzheimer's Disease, World Clinical Drugs, 2010.

[20] U. Preuss, J. Wong, and G. Koller, "Treatment of behavioral and psychological symptoms of dementia: a systematic review," Psychiatria Polska, vol. 50, no. 4, pp. 679-715, 2016.

[21] M. L. Levy, J. L. Cummings, L. A. Fairbanks et al., "Apathy is not depression," The Journal of Neuropsychiatry and Clinical Neurosciences, vol. 10, no. 3, pp. 314-319, 1998.

[22] A. D. Baharudin, N. C. Din, P. Subramaniam, and R. Razali, "The associations between behavioral-psychological symptoms of dementia (BPSD) and coping strategy, burden of care and personality style among low-income caregivers of patients with dementia," BMC Public Health, vol. 19, no. S4, p. 447, 2019.

[23] C. G. Ballard, C. L. Bannister, A. Patel et al., "Classification of psychotic symptoms in dementia sufferers," Acta Psychiatrica Scandinavica, vol. 92, no. 1, pp. 63-68, 1995.

[24] M. Gothert, "Serotonin discovery and stepwise disclosure of 5HT receptor complexity over four decades. Part I. general 
background and discovery of serotonin as a basis for 5-HT receptor identification," Pharmacological Reports, vol. 65, no. 4, pp. 771-786, 2013.

[25] S. G. Hasselbalch, K. Madsen, C. Svarer et al., "Reduced 5HT2A receptor binding in patients with mild cognitive impairment," Neurobiology of Aging, vol. 29, no. 12, pp. 1830-1838, 2008.

[26] Y. Yabuki, K. Matsuo, K. Hirano, Y. Shinoda, S. Moriguchi, and K. Fukunaga, "Combined memantine and donepezil treatment improves behavioral and psychological symptoms of dementia-like behaviors in olfactory bulbectomized mice," Pharmacology, vol. 99, no. 3-4, pp. 160-171, 2017.

[27] M. Flirski, T. Sobow, and I. Kloszewska, "Behavioural genetics of Alzheimer's disease: a comprehensive review," Archives of Medical Science, vol. 7, pp. 195-210, 2011.

[28] C. Holmes, M. Arranz, D. Collier, J. Powell, and S. Lovestone, "Depression in Alzheimer's disease: the effect of serotonin receptor gene variation," American Journal of Medical Genetics. Part B, Neuropsychiatric Genetics, vol. 119B, no. 1, pp. 40-43, 2003.

[29] T. Ueki, K. Mizoguchi, T. Yamaguchi et al., "Yokukansan, a traditional Japanese medicine, decreases head-twitch behaviors and serotonin $2 \mathrm{~A}$ receptors in the prefrontal cortex of isolation-stressed mice," Journal of ethnopharmacology, vol. 166, pp. 23-30, 2015.

[30] S. Shimizu, T. Tanaka, T. Takeda, M. Tohyama, and S. Miyata, "The Kampo medicine Yokukansan decreases microRNA-18 expression and recovers glucocorticoid receptors protein expression in the hypothalamus of stressed mice," BioMed Research International, vol. 2015, Article ID 797280, 8 pages, 2015.

[31] L. A. Pockros, N. S. Pentkowski, S. M. Conway, T. E. Ullman, K. R. Zwick, and J. L. Neisewander, "5-HT2A receptor blockade and 5-HT2C receptor activation interact to reduce cocaine hyperlocomotion and Fos protein expression in the caudateputamen," Synapse, vol. 66, no. 12, pp. 989-1001, 2012.

[32] K. Park, S. Lee, J. E. Yang, T. Song, and G. R. S. Hong, "A systematic review and meta-analysis on the effect of reminiscence therapy for people with dementia," International Psychogeriatrics, vol. 31, no. 11, pp. 1581-1597, 2019.

[33] F. Harrison, L. Aerts, and H. Brodaty, "Apathy in dementia: systematic review of recent evidence on pharmacological treatments," Current Psychiatry Reports, vol. 18, no. 11, p. 103, 2016.

[34] A. Anand, P. Khurana, J. Chawla, N. Sharma, and N. Khurana, "Emerging treatments for the behavioral and psychological symptoms of dementia," CNS Spectrums, vol. 23, no. 6, pp. 361-369, 2018.

[35] T. Ho, B. G. Pollock, B. H. Mulsant et al., "R- and S-citalopram concentrations have differential effects on neuropsychiatric scores in elders with dementia and agitation," British Journal of Clinical Pharmacology, vol. 82, no. 3, pp. 784-792, 2016.

[36] L. J. Bessey and A. Walaszek, "Management of behavioral and psychological symptoms of dementia," Current Psychiatry Reports, vol. 21, no. 8, p. 66, 2019.

[37] K. Yao, Y. F. Zhao, and H. B. Zu, "Melatonin receptor stimulation by agomelatine prevents $\mathrm{A} \beta$-induced tau phosphorylation and oxidative damage in PC12 cells," Drug Design, Development and Therapy, vol. 13, pp. 387-396, 2019.

[38] S. Inanir, U. S. Copoglu, H. Kokacya, R. Dokuyucu, O. Erbas, and A. Inanir, "Agomelatine protection in an LPS-induced psychosis-relevant behavior model," Medical Science Monitor, vol. 21, pp. 3834-3839, 2015.

[39] M. Mathys, "Pharmacologic management of behavioral and psychological symptoms of major neurocognitive disorder," Mental Health Clinician, vol. 8, no. 6, pp. 284-293, 2018.

[40] P. Holm, A. Ettrup, A. B. Klein et al., "Plaque deposition dependent decrease in 5-HT2A serotonin receptor in A $\beta$ PPswe/PS1dE9 amyloid overexpressing mice," Journal of Alzheimer's Disease, vol. 20, no. 4, pp. 1201-1213, 2010.

[41] C. L. Busceti, P. D. Pietro, B. Riozzi et al., " $5-\mathrm{HT}_{2 \mathrm{C}}$ serotonin receptor blockade prevents tau protein hyperphosphorylation and corrects the defect in hippocampal synaptic plasticity caused by a combination of environmental stressors in mice," Pharmacological Research, vol. 99, pp. 258-268, 2015.

[42] R. Hornedo-Ortega, G. da Costa, A. B. Cerezo, A. M. Troncoso, T. Richard, and M. C. Garcia-Parrilla, "In vitro effects of serotonin, melatonin, and other related indole compounds on Amyloid- $\beta$ kinetics and neuroprotection," Molecular Nutrition \& Food Research, vol. 62, no. 3, article 1700383, 2018.

[43] R. Heun, A. Ahokas, P. Boyer et al., "The efficacy of agomelatine in elderly patients with recurrent major depressive disorder," The Journal of Clinical Psychiatry, vol. 74, no. 6, pp. 587-594, 2013.

[44] G. Laux, B. Barthel, G. Hajak, M. Lemke, and H. P. Volz, "Pooled analysis of four non-interventional studies: effectiveness and tolerability of the antidepressant agomelatine in daily practice," Advances in Therapy, vol. 34, no. 4, pp. 895-914, 2017.

[45] Z. Bao-Yue, P. Xiao-Cong, J. Hao, W. Zhe, L. Ai-Lin, and D. Guan-Hua, Repositioning Drug Discovery for Alzheimer's Disease Based on Global Marketed Drug Data, Acta Pharmaceutica Sinica, 2019.

[46] V. Altinyazar and N. Kiylioglu, "Insomnia and dementia: is agomelatine treatment helpful? Case report and review of the literature," Therapeutic Advances in Psychopharmacology, vol. 6, no. 4, pp. 263-268, 2016.

[47] A. F. Carvalho, M. S. Sharma, A. R. Brunoni, E. Vieta, and G. A. Fava, "The safety, tolerability and risks associated with the use of newer generation antidepressant drugs: a critical review of the literature," Psychotherapy and Psychosomatics, vol. 85 , no. 5, pp. 270-288, 2016. 